\title{
MedienPädagogik
}

Zeitschrift für Theorie und Praxis der Medienbildung

\section{Zwischen Unsicherheit und Stabilisierung}

\section{Zur produktiven Wirkmächtigkeit von interaktionalen Artefakten, Störungen und Krisen im Kontext dis/ ability}

\author{
Marvin Sieger ${ }^{1}$ \\ ${ }^{1}$ Leibniz Universität Hannover
}

\section{Zusammenfassung}

Im Rahmen des vom BMBF geförderten Projekts be_smart werden die Teilhabepotenziale für Jugendliche und junge Erwachsene mit Behinderungserfahrungen im Rahmen von Musiziersituationen mit digitalen Geräten und Musik-Apps ausgelotet. Da es sich um komplexe sozio-technische und sozio-materiale Settings handelt, kann es innerhalb dieser Musiziersituationen vermehrt zu «Störungen» oder auch «Krisen» kommen. Um eine möglichst differenzierte Analyseoptik zu etablieren, wird innerhalb des Beitrags zunächst begrifflich in die Trias «Artefakt», "Störung» und "Krise» eingeführt. Für den Forschungszugang gilt aber, dass diese Analyseoptik nicht einfach dem Datenmaterial vorangestellt wird, sondern sie anhand der ständigen Auseinandersetzung mit dem empirischen Datenmaterial entsteht. Im Kontext dieser Trias wird deshalb des Weiteren ein Analyseraster für audio-visuelle Daten vorgestellt, das dabei helfen soll, die besagten Phänomene empirisch zu grundieren. Dabei soll «Komplexe» bzw. «Schwere Behinderung» vornehmlich als dis/ability analysiert werden, also als interaktionaler Prozess, in dem abilities und disabilities dynamisch fluktuieren. 


\title{
Between Uncertainty and Resilience. On the Productiveness of Interactional Artefacts, Disruption and Crisis in the Context of Dis/Ability
}

\begin{abstract}
In the context of the BMBF funded project be_smart («Importance of specific music Apps for participation of young people with complex disabilities in cultural education») audio-visual data of twelve self-conducted musicking sessions with young adults in the context of dis/abilities was obtained. The article starts with a definition and differentiation of the terms «artefact», "perturbation》 and «crisis》 in order to conceptualize moments of interactional uncertainty. The three terms are grounded in the analysis of audio-visual data. For the matter of empirical analysis of audio-visual data the article introduces a matrix which was crucial in order to structure, analyze and showcase such data. The matrix is instrumental especially for complex analysis of socio-technical settings in the context of dis/ ability.
\end{abstract}

\section{Einleitung}

Im Kontext «komplexe Behinderung» ${ }^{1}$ wurde im vom Bundesministerium für Bildung und Forschung (BMBF) finanzierten Projekt be_smart (Bedeutung spezifischer Musik-Apps für die Teilhabe von Jugendlichen und jungen Erwachsenen mit komplexen Behinderungen an kultureller Bildung) audio-visuelles Material von zwölf eigens durchgeführten Musiziersessions erhoben. Um des recht umfangreichen Datenkorpus (rund 20 Stunden audio-visuelles Material) Herr zu werden, wurde ein Analyserater für audio-visuelles Material entwickelt. Darüber hinaus wurden innerhalb dieses Rahmens die für die Analyse zentralen Begriffe «Artefakt», «Störung» und «Krise» entwickelt, die im Folgenden konzeptualisiert werden sollen.

1 Hier sind vornehmlich Menschen gemeint, bei denen z. B. dauerhaft (Zerebralund/oder Tetra-) Paresen und Spasmen auftreten. Diese Menschen sitzen oft in einem Rollstuhl oder nutzen eine Stehhilfe und Systeme zur unterstützenden Kommunikation, da sie oft keine «Verbalsprecher*innen» sind. 
Im Zusammenhang mit diesem Beitrag soll «Ästhetik» als eine (kontingente) Form der Wahrnehmung aufgefasst werden. Komplementär dazu soll «Macht» - relational - als eine Form bzw. eine Modalität der Kommunikation verstanden werden. Weiter wird davon ausgegangen, dass beide Bezugsgrössen - Ästhetik und Macht - sich ko-konstituieren und dass Subjekte und Objekte in «Machtbeziehungen verstrickt sind, in denen sie sich erst bilden» (Kämpf 2018, 159). Wir tun dies, um die These vorzubereiten, dass es im Kontext dis/ability ${ }^{2}$ vermehrt zu «Wahrnehmungskrisen» sowie zu «Machtasymmetrien» kommen kann. Diese Phänomene - so der hier eingenommene Blickwinkel - werden vornehmlich in situ, durch «Körper» bzw. durch Leiber - aufgefasst als sensorische Erfahrungs- und Gefühlsräume (vgl. Schmitz 1969) - und «Objekte» (z. B. digitale Geräte, Rollstühle usw.) konstituiert. Sie entfalten ihre Wirkmächtigkeit auf der Teilnehmendenebene zumeist unterhalb der Schwelle des bewusst und reflexiv Wahrnehmbaren. Um diese Phänomene «sichtbar» zu machen, treten wir dafür ein, Analysen von audio-visuell konservierten Interaktionssituationen zu forcieren. Aus dieser Perspektive wird dis/ability als eine bestimmte Form der Wahrnehmung sowie als eine bestimmte Modalität der Kommunikation innerhalb eines (sozio-materialen) Netzwerks bzw. innerhalb eines Ensembles unterschiedlicher Entitäten, die es jeweils situativ zu bestimmen und zu analysieren gilt. Anhand der Phänomene «Artefakt», «Störung» und «Krise» - so die Stossrichtung - kann man diese Formen (z. B. in Gestalt interaktionaler/kommunikativer Asymmetrien) analytisch einkreisen.

2 Dis/ability wird hier als analytischer und methodologischer Begriff eingesetzt, der seinen Gegenstand als dynamisch-fluides und auszuhandelndes Produkt von Aktantenbeziehungen versteht. Für diesen Beitrag wird somit weniger von Komplexer oder Schwerer Behinderung gesprochen, sondern vornehmlich von dis/ability. Wer wann etwas kann oder nicht kann, wer beeinträchtigt ist, hängt somit erst einmal ausschliesslich von bestimmten situativen Stabilisierungen und sozio-technischen Beziehungen in einem soziomaterialen Netzwerk ab. 


\section{Artefakte, Störungen, Krisen}

\section{$1.1 \quad$ Artefakte}

Wir gehen mit Goffman davon aus, dass Unsicherheiten und ihre interaktionale Bearbeitung der produktive Normalfall jeder Interaktionsordnung (1967) sind. Mit dem Begriff Artefakt sind im deutschen Sprachraum grundsätzlich zwei unterschiedliche Phänomenbereiche aufgerufen. Der erste Bereich (Typ I) bezieht sich - angelehnt an die lateinische Herkunft des Wortes - auf einen von Menschen hergestellten dinglich-materiellen Gegentand, auf ein intendiertes und praktisch hergestelltes «um-zu»-Produkt menschlicher Praxis. ${ }^{3}$ Der zweite Bereich (Typ II) umfasst ein Phänomen, das vor allem dann auftritt, wenn komplexe und mehrschrittige (technische) Verfahrensweisen zur Anwendung gebracht werden. So wird z. B. in der modernen medizinischen Diagnostik ein Artefakt als ein nicht intendierter und ungewollt herbeigeführter (Neben)Effekt einer wissenschaftlich-technischen Verfahrensweise aufgefasst, der den eigentlichen diagnostischen Erkenntnisgewinn blockiert. Bei bildgebenden Verfahren manifestiert sich ein Artefakt beispielsweise als ein konkret morphologisch-visuelles Phänomen - z. B. als luftbedingter «Subtraktions- oder Additionseffekt», der entweder bei zweidimensionalen Verfahren als Verschattung oder Aufhellung auftritt - ohne jeglichen oder gar mit irreführendem Erkenntnisgewinn. Entscheidend ist, dass ein Artefakt hier - wie auch in den Informationswissenschaften, der Statistik und anderen Lebenswissenschaften - negativ konnotiert ist. ${ }^{4}$ Artefakte sind hier - wenn man so möchte - auf komplexe Vorgänge zurückgehende Metaphänomene, Residuale, die auftauchen oder emergieren, wenn technische Verfahrensweisen methodisch gebildet und zur Anwendung gebracht werden. Letztlich wird das Auftreten von Artefakten innerhalb dieses Phänomenbereichs drei unterschiedlichen Ursachenkomplexen zugerechnet: (1) Dem

3 https://www.duden.de/rechtschreibung/Artefakt. Letzter Abruf: 4. Mai, 2021.

4 Man trachtet also mehr oder minder - implizit wie explizit - danach, Artefakte zu vermeiden, oder sie eben bei Auftreten zu neutralisieren. Sie haben - wie bereits gesagt - keinen Erkenntniswert (anders als bspw. in der Kunst). Sie sind primär undesired and unintended aberrations in a data processing context. 
jeweiligen wissenschaftlichen Verfahren/der Methode selbst (Validität). (2) Den materialen und temporalen Bedingungen (dem Substrat, den Komponenten, der Datengüte) eines Verfahrens bzw. einer Methode (Reliabilität). (3) Der menschlichen Unzulänglichkeit (Objektivität). Innerhalb der Sozialwissenschaften spielen ebenfalls beide Typen eine Rolle. Der erste Typ ist kultursoziologisch/anthropologisch gerahmt und fokussiert auf die Rolle von hergestellten Gegenständen mit Blick auf Verständigungs- und Akkulturationsprozesse. Der zweite Typ zielt auf nomologisch und quantitativ verfahrende (empirische) Zugänge zur sozialen Wirklichkeit. Hier wird anhand eines Artefakts definiert, «inwieweit die Ergebnisse empirischer Sozialforschung die gültige Widerspiegelung sozialer Realität darstellen» (Hilgers 1997).

Ich möchte hingegen den Begriff «Artefakt» für einen dritten Typus von Phänomenen (Typ III) in Anspruch nehmen, nämlich für nicht intendierte Effekte innerhalb relationaler und interaktionaler Verschränkungszusammenhänge, die organisch-körperliche und leibliche sowie soziale und medial-dingliche Komplexitäten betreffen. Demnach ist ein Artefakt ein Phänomen, das innerhalb von Interkationen zwar «sichtbar/wahrnehmbar» (leiblich-körperlich) auftaucht, aber keine besonderen (bewussten) Mittel, Massnahmen oder Anstrengungen der Interaktionsteilnehmenden erfordert, damit die Interaktion weiter fortgesetzt werden kann. Es ist aber nicht so, dass diese Artefakte eigentlich keine Rolle innerhalb einer Interaktionssituation spielten, vielmehr werden diese Artefakte von uns als (produktive) (Mit-)Konstituenten der betreffenden Interaktionssituationen aufgefasst. Sie verleihen ihnen - wenn man so möchte - einen eigenen, temporalen, sozialen und sachlichen Charakter und spannen eigene Referenzräume auf. Es handelt sich hierbei um nicht intendierte Effekte, die zumeist mit einer bestimmten (Körper-)Aktivität auftreten.

$\mathrm{Zu}$ nennen sind hier insbesondere für unseren Forschungskontext: Spasmen, Zuckungen, (nicht intentionales) Lautieren usw. Für dis/abilty gilt, dass diese Phänomene u. U. vermehrt und ggf. mit starker Intensität auftauchen können. Soziologisch gesehen kann man diese Phänomene insbesondere für das menschliche Gesicht - als ein teilweise ausbleibendes Facework charakterisieren. Facework kann nach Goffman verstanden werden als «the actions taken by a person to make whatever he [*she] is doing 
consistent with face» $(1956,216) .{ }^{5}$ Diese «Inkonsistenz» in der Darstellung von (an)zeigendem oder sprachlichem (indexikalem) Ausdruck auf der einen, Mimik/Körperausdruck auf der anderen Seite - nach Bateson (1969) «double bind» genannt - wird in diesen Interaktionszusammenhängen in der Regel durch Gesten, Features von Sprachein- und -ausgabegeräten (Talker), Lautieren, Assistenz etc. kompensiert.

Innerhalb des Forschungskontextes be_smart spielen aber auch die beiden Typen I und II eine Rolle. Artefakte im Sinne von Typ I (Objektebene) kamen z. B. in Form digitaler Geräte zum Einsatz. Forschungslogisch ging es uns darum, die Rolle dieser Geräte mit Blick auf den Interaktionsverlauf zu analysieren. Es kamen aber eben auch Artefakte im Sinne von Typ II (Operationsebene) zum Tragen, nämlich dann, wenn im Rahmen der Musiziersessions auch ein kleiner Interviewteil zum Musiknutzungsverhalten der Teilnehmenden zustande kam. Da die meisten der Interviewees über sogenannte Talker ${ }^{6}$ kommunizieren, kam es während der Interviews teilweise zu recht langen Antwortlatenzphasen (je nach Interviewee und eingesetzter Technik kann die Antwort auf eine Frage mehrere Minuten dauern). Die eigentlichen Artefakte entstanden dann allerdings durch den verbal sprechenden Interviewer, und zwar dadurch, dass diese auftretenden «Sinnlücken» von ihm nicht intendiert verbalsprachlich (mit Text) gefüllt wurden. Dies mag auch mit dem bereits beschriebenen Ausbleiben von Facework zu tun haben. Dieses Ausbleiben kann Verbalsprecher dazu anregen, die Interaktion phonetisch-syntaktisch «zweitzukodieren».

Für die im Forschungskontext realisierte empirische Analyse gilt aber, dass die Ebene der Körper und Objekte mit Blick auf die Analyse von Artefakten zentral ist (dies gilt insbesondere für Typ I und III). Artefakte sind im Rahmen unserer Überlegungen (anders als in der bereits vorgestellten Artefaktauffassung von Typ II) in erster Linie produktive Körper- und Leibphänomene. Für den weiteren Fortgang des Beitrags ist danach zu fragen, inwieweit diese Artefakte (Typ III) Störungspotenzial aufweisen und

5 Menschen/Personen haben aus dieser Sicht für unterschiedliche Situationen je unterschiedliche Faces im Repertoire.

6 Ein Talker ist technisch gesehen ein (elektronisch unterstütztes) Text-Eingabe/Auditive-Ausgabe System. Ein prominenter Talkernutzer ist der verstorbene Astrophysiker Stephen Hawking. 
inwieweit Störungen in Interaktionszusammenhängen als interaktional produktive Momente oder eben als die Interaktion inhibierende Momente aufgefasst werden können.

\subsection{Störungen}

Störungen werden aus sozialwissenschaftlicher Sicht vornehmlich dem Zuständigkeitsbereich der Psychologie überlassen. ${ }^{7}$ Der wissenschaftskommunikative und somit in diesem Feld auch praktisch-diagnostische Output ist enorm (siehe bspw. das DSM-5 der APA). Innerhalb der Soziologie kommt dem Begriff «Störung» im Rahmen kybernetisch informierter soziologischer Systemtheorie zwar eine gewisse Bedeutung zu, basal gesehen geht es aber erst einmal nicht darum, wie (und wo) «Störungen» auftreten und bearbeitet werden können, ${ }^{9}$ sondern um die Frage, wie «Verstehen» unter Bedingungen der wechselseitigen Intransparenz (von Kommunikationsteilnehmenden oder eben Systemen) überhaupt möglich wird. Mit anderen Worten: «Störungen» sind im Sinne des Theoriekanons als konstitutive Bedingungen mit Blick auf die Operationalität sozialer und psychischer Systeme angelegt. Dabei ist zu berücksichtigen, dass aus Perspektive der soziologischen Systemtheorie «Verstehen» letztlich keine zwingende Bestandsvoraussetzung sozialer Systeme ist (genauso wenig wie «Körper» oder «Objekte», die in die Umwelt des Sozialen bugsiert werden). Insofern zielt auch der Analysefokus, sobald Störungen angenommen werden, vornehmlich auf die Ebenen «Organisationen»(Baecker 2011), «Funktionssysteme», oder eben «Gesellschaft» (Luhmann 1997) ab. Dabei wird vom «order from noise» Prinzip ausgegangen (von Foerster 2003), also davon, dass ausschliesslich das betreffende System selbst seine (eigenen) Störungen entstören kann, es also Perturbationen durch die Elemente

7 «Störungen» werden hier zumeist Individuen zugeschrieben (sie sind entweder gestört oder stören). Die ko-konstitutive Funktion der zuschreibenden Instanz und die Materialität dieses Prozesses bleiben dabei zumeist unterbelichtet.

8 https://www.psychiatry.org/psychiatrists/practice/dsm.

9 Es sei denn in der Nische der Interventionstheorie, die therapeutisch konnotiert ist (siehe dazu Willke 1994). Mir geht es im Zusammenhang mit Störungen (und Krisen) explizit nicht um therapeutische Implikation bzw. einen therapeutischen Anspruch. 
reguliert, die es selbst herstellt und selegiert. Die Störungsbearbeitung wird durch die «intrinsic structural properties» (ebd. 2003, 13) der Elemente (bzw. durch deren Relationierungen) selbst vollzogen. Groenemeyer bemerkt in diesem Kontext:

«Die soziologische Diagnose gesellschaftlicher Störungen und sozialer Probleme setzt immer auch Annahmen über einen (ungestörten〉 oder 〈normalen〉 Zustand der Gesellschaft voraus.» (1999, 14)

Für die soziologische Systemtheorie gilt aber grundsätzlich, dass «Irritation» ${ }^{10}$, «Perturbation», «noise», «Störung》, «Rauschen» usw. Bestandvoraussetzung sozialer und psychischer Systeme sind. Mit anderen Worten: Ohne Störungen keine Systemevolution! Alle Störungen und Irritationen können aber eben nur in Grenzen verarbeitet werden. Gansel und Ächtler bemerken in diesem Zusammenhang:

«Gesellschaftssysteme erneuern sich beständig gerade auch in der Auseinandersetzung mit ihren anthropologischen, soziopolitischen, technisch-ökonomischen, ökologischen oder auch kulturellen «Störfällen〉.» $(2013,9)$

Nach der Kommunikationsforschung und der linguistischen Theorie liegt hingegen eine Störung vor, wenn beispielsweise die Verwendung eines Ausdrucks im laufenden Gespräch von einer Person nicht verstanden (bzw. hinterfragt) wird und Reformulierungshandlungen vollzogen werden. Dieser pragmatische Aushandlungsprozesses wird mitunter als «Störungsphase» begriffen in der

«[...] Bearbeitungen, Erläuterungen und Paraphrasen der in der Kommunikation behandelten Inhalte statt[finden], da erst ein gemeinsam geteilter Sinnhorizont hergestellt werden muss.» (Apel et al. 2013, 108f.)

10 Die unterschiedlichen Akzentuierungen dieser Begriffe und Konzepte können im Rahmen dieses Beitrags nicht herausgearbeitet werden. Irritation im systemtheoretischen Sinne kann jedenfalls aufgefasst werden als systemevolutives und «konstruktives Prinzip, das systemischen Differenzierungs- und Adaptionsprozessen überhaupt zugrunde liegt und dauerhaften Anlass für systemische Kommunikation wie Selbstbeobachtung und Selbstbeschreibung bietet» (Gansel und Ächtler 2013, 10). 
Medientheoretisch gesprochen kann Störung dann

«[...] jeder Zustand im Verlauf einer Kommunikation heissen, der bewirkt, dass ein Medium (operativ) seine Transparenz verliert und in seiner Materialität wahrgenommen wird, und Transparenz jeder Zustand, in dem die Kommunikation nicht 〈gestört〉 ist, also das Medium als Medium nicht im Fokus der Aufmerksamkeit steht.» (Jäger 2004, 62 zitiert nach Gansel 2013, 39)

Anschlussfähig ist hier die von Heidegger eingebrachte Unterscheidung von Vorhandenheit und Zuhandenheit (1953, 102). Diese Unterscheidung kann mit Blick auf die beiden analytischen Bezugsgrössen (technische) «Geräte» und «Körper» verdeutlichen, dass zumeist die eine Bezugsgrösse zugunsten der jeweils anderen in den Hintergrund tritt. Somit ist Körper jeweils vorhanden wenn er (a) entweder direkt für ein Gegenüber als Zeichenprozessor fungiert (Anzeigen/Referenzieren) oder er (b) bestimmte Affektnormen unterläuft (Spasmen/Lautieren etc.). Umgekehrt ist er zuhanden, wenn er phänomenologisch gesprochen nicht als «Objekt» für einen Beobachter gesetzt ist, also nicht mehr in Selbstreferenz (Alter verweist auf «seinen» Körper) oder Fremdreferenz (Ego verweist auf den Körper des Gegenübers) eindeutig von einer Aktivität diskriminierbar vorhanden ist. Das gleiche gilt für ein (technisches) Gerät: Es ist vorhanden, wenn es z. B. streikt oder nicht reibungslos im Interaktionsgeschehen aufgeht. Bei diesen «Stör- und Unfällen» wird dann die «[...] Medialität [und Materialität] von Medien sichtbar.» (Gansel und Ächtler 2013, 8). Zuhanden ist ein Gerät hingegen, wenn es im Modus des Hantierens quasi die Schwelle des «Entitären», Einzelnen, Für-sich-genommenen und Geschiedenen unterläuft. Bergmann konstatiert im Rahmen von Interaktionsstörungen:

«Potentielle Störungen führen nicht automatisch zur Unterbrechung einer laufenden Interaktion. Sie evozieren Relevanz-, Aufmerksamkeits- und Kontinuitätskonflikte [...]. Nicht das 〈störende Ereignis〉 ist schon die Unterbrechung, sondern erst die Unterbrechung macht aus einem konkurrierenden Ereignis eine Störung.» (Bergmann 2012, 6) 
Ich möchte an Bergmann anschliessen und Störungen folgendermassen konzeptualisieren: Eine Störung ist ein Phänomen, das die Interaktionsteilnehmenden in der Regel dazu zwingt, Aufmerksamkeit bzw. Ressourcen (Routinen) zur Bearbeitung der Störung zu mobilisieren. ${ }^{11}$ Dabei spielen dann unterschiedliche Bezugsmodi, Medien und Mittel eine Rolle, die zur (meist unmittelbaren) Bearbeitung/Lösung der Störung beitragen. Störungen sind in der Regel antizipierbar in dem Sinne, dass allen Beteiligten klar ist, dass sie jederzeit spontan auftauchen (emergieren) können. Deswegen haben Interaktionsteilnehmende in der Regel ein Repertoire an Routinen an der Hand, die es ihnen ermöglichen, Störungen unmittelbar zu bearbeiten. Darüber hinaus gilt: Störungen müssen als solche wahrgenommen bzw. (analytisch) identifiziert werden, damit sie auf das Display eines Interaktionszusammenhangs kommen können. In unserem Forschungskontext verweisen Störungen immer auf Handlungsträgerschaft. Allerdings darf man hier keinen verkürzten Handlungsbegriff zugrunde legen. «Handlungsträgerschaft» soll eben nicht an einzelnen Individuen und ihren (intendierten) Aktivitäten festgemacht werden.

Fällt beispielsweise ein digitales Musizierinstrument aus (Störung), zeigt dieser Vorfall immer mit an, dass diese Geräte in (soziale) Unternehmungen und Zwecksetzungen eingespannt sind, die über sie selbst (Netzwerke) und die beteiligten Individuen (oder Handlenden) hinausweisen. Die primäre Referenz mit Blick auf die Analyseebene «Störung» ist im hier vorgestellten Forschungskontext die des (technischen) «Objekts». Daran anknüpfend kann man einräumen, dass mit Blick auf die analysierten Interaktionszusammenhänge die auftretenden Störungen nicht selten produktive Momente waren, da sie durch ihren Ausfall eben auch ihre Wichtigkeit für das Zustandekommen und Erhalten der Interaktionsordnung indizierten und sie darüber hinaus die Interaktionsteilnehmenden dazu zwangen, gemeinsam einen Weg zu entwickeln, die Interaktion fortzusetzen.

11 Bis zu einem gewissen Niveau lassen sich Störungen wohl ignorieren. Werden sie aber vollständig ignoriert, ziehen sie in der Regel die baldige Beendigung eines bereits stabilisierten Interaktionszusammenhangs nach sich. 


\subsection{Krisen}

Die meisten gängigen sozialwissenschaftlichen Krisendefinitionen lassen Interaktionskrisen weitgehend ausser Acht. Krisen sind demnach ausschliesslich auf Ebene von Diskursen und Gesellschaft sozialwissenschaftlich relevante Tatbestände. So z. B. Steg:

«Um den Krisenbegriff als soziologische bzw. sozialwissenschaftliche Analysekategorie adäquat verwenden zu können, muss [...] an einem wissenschaftlich-analytischen Begriff festgehalten werden, der nicht für individuelle und psychologische, sondern ausschliesslich für gesamtgesellschaftlich relevante Phänomene aussagekräftig ist.» $(2020,431)$.

Einen Gegenpol dazu nehmen Positionen ein, die die vielbeschworene «Krise des Subjekts» (Spitta 2018, 226) ausrufen. ${ }^{12}$ Dewey und Mead entwerfen aus der pragmatischen Tradition einen anderen Zugang. Sie betrachten «Krisen» als Suppositionen für «reflexives Handeln». Dazu bemerken Antony, Sebald und Adloff:

«Im Hinblick auf Handlungs- und Interaktionskrisen [fungiert] [...] das Problematischwerden des primären Weltbezugs, d. h. eine wie auch immer geartete 〈Störung〉 habitueller Handlungskoordination, als Bedingung der Möglichkeit des Auftretens reflexiver Operationen [...]. Reflexives Handeln hat seinen Ursprung in der situativen Inadäquanz gewohnheitsmässigen Handelns und dient der Bewältigung von Handlungsproblemen.» $(2016,4)$

Ich möchte hingegen betonen, dass Störungen nicht unbedingt «reflexives Handeln» (was immer dies auch genau sein mag) affizieren. ${ }^{13}$ Erst sobald eine Störung zur Krise wird - wenn gemeinsame Praxiskoordination aufgrund einer Disruption nicht mehr möglich ist, die sequenzielle Ordnung, der Ablauf einem zu harten Schock ausgesetzt wurde -, veranlasst dieser Zustand durch unmittelbar wahrnehmbare Krisenhaftigkeit, durch die Absenz von Sinn, Reflexion. Das heisst, die Krise wird so erst objektiviert, vergegenständlicht und für Denk- und kommunikative Operationen

12 Die «Krise des Subjekts» ist immer gleichzeitig auch «die Krise des Objekts».

$13 \mathrm{Zu}$ einer plausiblen Kritik des Konzepts «reflexives Handeln» siehe Schatzki 2016. 
«anschliessbar» und so mit Sinn angereichert. Aus dieser Perspektive erzeugt erst die Koppelung von Wahrnehmung, Bewusstsein und Kommunikation eine Krise. Damit sind Krisen auf Interaktionsebene Ohnmachtsphänomene, denn die Situation entzieht sich im Krisenfall gänzlich der Steuerung durch die Interaktionsteilnehmenden. Des Weiteren kann es auch sein, dass Ressourcen - bzw. Mittel, Routinen, (technische) Objekte, knowing how etc. - in der Interaktion (aus welchen Gründen auch immer) nicht erreichbar sind. Mit Blick auf das Phänomen Macht lässt sich aus diesem Blickwinkel sagen: Macht kann erst dann wieder kanalisiert werden, wenn die Krise - mit welchen Mitteln auch immer - (gemeinsam) überwunden wurde. Kommt es aber zu punktuellen Überschüssen, zu ausgeprägten Machtasymmetrien, führen diese zumeist wieder zurück in die Krise. Aus dieser Perspektive sind Werte wie «Freiheit», «Autonomie», «Handlungsfähigkeit», oder «Subjektivität» Produkte von Machtbeziehungen (vgl. Saar 2017, 158). Macht wird dann nicht im Sinne einer fremdreferenziellen Handlungsoktroyierung (wie z. B. bei Mannheim 1950), sondern als durch eine in einem Netzwerk konstituierte Grösse aufgefasst, in welchem sie fluktuiert. Sie wird zu einem relational- und interaktionalen Phänomen, das sich aus den praktischen Verbindungen und den (Un)Möglichkeiten ergibt, die die beteiligten Aktanten im Interaktionsverlauf realisieren. Ein Aktant im Sinne Latours (2008) kann als eine Entität bzw. als eine Relation aufgefasst werden, die in Interaktionen einen Unterschied macht. Explizit eingeklammert sind dabei (technisch-digitale) Artefakte bzw. soma-dingliche Relationen.

Krisen vermögen es, die (unmittelbare) Zukunft des Interaktionssystems kontingent zu setzen und somit gleichsam Machtbeziehungen punktuell ausser Kraft zu setzen. Darin liegt Chance und Gefahr zugleich. Ähnlich wie für Störungen gilt analytisch gesehen auch für Krisen:

«Das Interesse liegt auf den körperlichen, auch sensuellen, Kompetenzen der Akteur/innen und dem Fragwürdig-werden ebendieser.» (Krämer 2016, 51)

Im Rahmen unseres Forschungsvorhabens liegt der Fokus stark auf der Frage, welche Funktion (technische) Objekte mit Blick auf Krisengenese und Prävention in komplexen sozio-technischen Ensembles einnehmen. 
Dabei sind Krisen «mehr als blosse minimale Störungen innerhalb des Handlungsverlaufs oder leichte Erschütterungen der Interaktionsgewohnheiten» (Krämer 2016, 37). In diesem Zusammenhang sehe ich es als äusserst produktiv an, eine zeigend-ethnografische Interaktionsforschung zu betreiben (Mohn 2013). Interaktionskrisen geraten dann «nicht mehr als reine Beobachtungsaggregate in den Blick, sondern erhalten als körperliche, situierte und selbst erlebte Situationen ihre empirische Präsenz» (Krämer 2016, 47).

Analytisch lässt sich für den hier vorgestellten Forschungskontext festhalten, dass zwei Typen von Interaktionskrisen unterschieden werden können: (1) Temporäre Krisen: hier kommt es zu interaktionalen Unterbrechungen, es müssen neue Mittel oder Wege mobilisiert werden, um die Krise zu bearbeiten und die Interaktion fortsetzen zu können. (2) Dauerhafte Krisen: Sie führen zur zeitstabilen Auflösung des betreffenden Interaktionssystems sowie zur Auflösung von auch bereits institutionalisierten Systemen.

\section{Entwicklung eines Analyserasters für audio-visuelles Material}

Da es sich gerade bei audio-visuellem Material in der Regel um sehr «dichte» Daten $^{14}$ handelt, gilt hier besonders, dass üblicherweise diverse Fragen am Material entstehen, die den «eigentlichen» Forschungskontext erst einmal überschreiten. Somit gilt es, einerseits die Komplexität zu reduzieren, die dieses Material mitunter bereitstellt, um die grundlegenden Forschungsfragen nicht aus dem Blick zu verlieren, andererseits aber offen zu bleiben für interessante Fragen, die am Material entstehen. Grundsätzlich ist die hier vorgestellte Vorgehensweise daran orientiert, «nicht rein sprachliche Konversationen, sondern umfassende soziale Situationen [...]» (Knoblauch

14 Geertz hat den Begriff «thick description» $(2017,11)$ für heterogene, ineinander verflochtene und reflexive ethnografische Beobachtungsheuristiken und Methoden verwendet. Für das hier vorgestellte Konzept zur Analyse audio-visueller Daten gilt ebenso, dass u.a. die Erzeugungshintergründe in die Analyse einbezogen werden und man daher von einer «dichten Beschreibung» (bzw. von einer «dichten Analyse») sprechen kann. Der (machtvolle) Aspekt des Erzeugungshintergrunds kann aber leider nicht im Rahmen dieses Beitrags expliziert werden. 
2004, 129) als «Grundeinheiten» für Interaktionsanalysen zugrunde zu legen. Im Rahmen des Erstellens eines Analyserasters für audio-visuelles Material gilt es - ausgehend von einer ersten Fragestellung (z. B. der Frage nach Interaktionskrisen) - bestimmte analytische Dimensionen bzw. «Mikrofragestellungen» zu generieren. In Projektkontexten mit extensivem Datenkorpus müssen dann unweigerlich drei Komplexitätsreduktionsmechanismen vollzogen werden, die die audio-visuelle Materialsammlung quasi erst zu einem Datenkorpus machen:

1. Es sollte eine spezifische Fragestellung bereits vor dem Erstellen von Transkripten/Partituren entworfen werden.

2. Das audio-visuelle Material muss in der Zeitdimension selegiert werden (begrenzte Auswahl besonders aussagekräftiger Sequenzen). Dabei darf die sequenzielle Verlaufslogik des konservierten Datenstücks nicht ignoriert werden.

3. Es muss eine Auswahl von zu transkribierenden Modalitäten getroffen werden (siehe dazu Heath et al. 2010, 12).

Im Folgenden wird ein tabellarisches Analyseraster mit zwei Beispielsequenzen präsentiert, das angelegt wird, nachdem die Schritte eins bis drei vollzogen sind. Das vorgestellte Analyseraster fokussiert auf das Aufkommen interaktionaler Krisenmomente.

In Spalte eins (Datenstück/Zeitmarken) sind die zu analysierenden/ bereits analysierten Sequenzen untereinander eingetragen. Jede Sequenz wird durch einen eigenen Dateinamen (Buchstaben), eine Dateiendung/ einen Dateityp und die Angabe eines Zeitintervalls repräsentiert. In der zweiten Spalte wird für jede Sequenz eine kurze Situationsbeschreibung (in der Regel wenige Sätze) angefertigt. Für unser Datenkorpus kamen Sequenzen von ca. 10 Sekunden bis zu 4 Minuten Länge zustande. Device (digitale Geräte, Spalte 3): Hier wird mit «zuhanden» oder «vorhanden» vermerkt, welchen Status das betreffende Device mit Blick auf das Interaktionsgeschehen innerhalb der jeweiligen Sequenz aufweist. 


\begin{tabular}{|c|c|c|c|c|c|}
\hline $\begin{array}{l}\text { Datenstück/ } \\
\text { Zeitmarken } \\
\text { (Sitzungs- } \\
\text { nummer, } \\
\text { Datum) }\end{array}$ & Kurze Situationsbeschreibung & Device & Körper & $\begin{array}{l}\text { Verbal- } \\
\text { sprache }\end{array}$ & $\begin{array}{c}\text { Externe } \\
\text { Komple- } \\
\text { xität }\end{array}$ \\
\hline $\begin{array}{l}\text { Datenstück } \\
\text { X.MP4: 4:19- } \\
7: 49\end{array}$ & \begin{tabular}{|l|} 
Es reihen sich mehrere Störungsereig- \\
nisse auf unterschiedlichen Ebenen \\
aneinander und verdichten sich letztlich \\
zu einer Krisis, die das (kollaborative) \\
Explorieren von Musik-Apps nicht wirklich \\
in Gang kommen lässt. Bei 4:19 entziehen \\
sich die Peripheriegeräte der Kopplung \\
per Bluetooth mit dem iPad. Zeitgleich \\
setzt der Interviewee seinen Talker ein \\
und talkert (zum wiederholten Male) «ich \\
habe eine Frage». Parallel dazu hat der \\
Vater einen aktiven und dominanten Part \\
im explorativen Interview übernommen. \\
Dies zeigt sich u.a. auch daran, dass der \\
Vater den rechten Arm des Interviewees \\
greift (01:21), um diesen zu disziplinieren \\
bzw. «fremdzusteuern».
\end{tabular} & $\begin{array}{c}\text { vorhan- } \\
\text { den }\end{array}$ & $\begin{array}{c}\text { vor- } \\
\text { handen } \\
\text { (Vater) }\end{array}$ & ja (Vater) & ja (Vater) \\
\hline $\begin{array}{l}\text { Datenstück } \\
\text { Y.MP4: 01:10- } \\
01: 57\end{array}$ & $\begin{array}{l}\text { Der Interviewee lotet die Möglichkeiten } \\
\text { der aufgerufenen Musik-App aus, verliert } \\
\text { aber schnell das Interesse und will meh- } \\
\text { rere Male den Tisch verlassen, wobei die } \\
\text { Mutter ihn (zunächst) mit vollem Körper- } \\
\text { einsatz davon abhält. }\end{array}$ & $\begin{array}{c}\text { vor- } \\
\text { handen }\end{array}$ & $\begin{array}{c}\text { vor- } \\
\text { handen } \\
\text { (Mutter) }\end{array}$ & $\begin{array}{l}\text { ja } \\
\text { (Mutter } \\
\text { und } \\
\text { Intervie- } \\
\text { wer) }\end{array}$ & $\begin{array}{c}\text { ja } \\
\text { (Mutter) }\end{array}$ \\
\hline
\end{tabular}

Tab. 1: Analyseraster zur Krisengenese.

Die Unterscheidung vorhanden/zuhanden soll gerade mit Blick auf die beiden analytischen Bezugsgrössen «Device» und «Körper» verdeutlichen, dass zumeist die eine Bezugsgrösse zugunsten der jeweils anderen in den Hintergrund tritt. Somit sind Körper jeweils vorhanden, wenn sie entweder (a) direkt als Zeichenprozessoren fungieren (Anzeigen/Referenzieren) oder (b) bestimmte Affektnormen unterlaufen (sich der intentionalen Steuerbarkeit entziehen). Umgekehrt sind sie zuhanden, wenn sie, phänomenologisch gesprochen, nicht als «Objekt» für einen Beobachter gesetzt sind, sie also nicht mehr in Selbstreferenz (Alter verweist auf «seinen» Körper) oder Fremdreferenz (Ego verweist auf den Körper des Gegenübers) eindeutig von einer Aktivität diskriminierbar vorhanden sind. Das gleiche gilt für ein Gerät: Es ist vorhanden, wenn es z. B. streikt oder nicht reibungslos 
im Interaktionsgeschehen aufgeht und es ist zuhanden, wenn es im Modus des Hantierens quasi die Schwelle des «Entitären》, Einzelnen und fürsich-genommenen unterläuft. Körper (Spalte 4): Hier gilt das gleiche wie für «Device», ein ja oder nein gibt an, ob Körper im Rahmen der Sequenz «vorhanden» oder «zuhanden» ist (siehe dazu auch Kapitel 2 - Störungen). Verbalsprache (Spalte 5): Hier wird lediglich mit jeweils ja oder nein angeführt, ob in der betreffenden Sequenz Verbalsprache vorkommt bzw. eine Rolle spielt (darunter fällt auch Lautieren). Externe Komplexität (Spalte 6): In unserem Datenkorpus lassen sich vermehrt Situationen (Sequenzen) ausweisen, in denen ungeplant bestimmte Protagonisten auf die Bühne getreten sind. Es gab Situationen, in denen die Interviews von signifikanten Anderen (z. B. von Eltern) zur Selbstinszenierung und Macht-/Rollendarstellung ausgenutzt wurden. Die Protagonisten haben sich dann quasi aus dem Off heraus sukzessive immer weiter in den Sicht-/Hörbereich der Kamera geschoben oder keine Rücksicht auf die geplante und stattfindende Musiziersession genommen, indem sie Pflegetätigkeiten vorbereitet und durchgeführt haben. Dieses Item dient noch einmal dezidiert dazu, fremdreferenziell induzierten Störungen (und Krisen) auf die Spur zu kommen.

Im Rahmen des Rasters ist die methodologische Überlegung zentral, eine Fragestellung nicht einfach dem Material voranzustellen bzw. auf das Material zu projizieren, sondern sie am Material (weiter) zu entwickeln und anhand von möglichst kurzen und prägnanten Sequenzen zu fundieren. Empirische Phänomene, theoretisches Vokabular ${ }^{15}$ und Konzepte gehen dann Hand in Hand und werden am Material vermittelt. Dabei ist ein iterativ-zyklisches Vorgehen gut geeignet (Glaser und Strauss 2017; Corbin und Strauss 2015; Strübing 2014; Breuer et al. 2019), also die prinzipiell offene sowie interdependente Anpassung von Fragestellung, Datum, Methode und theoretischer Generalisierung. Darüber hinaus votieren wir dafür, Echtzeitanalysesitzungen in Gruppensettings zu privilegieren, also das Material gerade für die Analyse von «social activities in real-time: talk, visible conduct [...] the use of tools, technologies, objects and artefacts» (Heath et al. 2010, 5f.) in seiner temporalen und modalen Eigenlogik (intersubjektiv) ernst zu nehmen und demnach auch den Analyseprozess diesem Modus anzupassen und Analyseprotokolle anzufertigen.

15 Wie z. B. die im Rahmen des Beitrags bereits erläuterten Begriffe «Artefakt», «Störung», «Krise». 
So dient das Analyseraster zum einen dazu, bestimmte Sequenzen dokumentarisch zu erfassen, sie tiefergreifend zu untersuchen und sie mehrere Male in ihrer jeweiligen sequenziellen Eigenlogik ${ }^{16} \mathrm{zu}$ betrachten. Zum anderen wird durch die Erfassung mehrerer Sequenzen ein Vergleichshorizont aufgespannt, der Dokumentation, Darstellung, Strukturierung und Relationierung der Daten in einem ist. Mit Blick auf die Phänomene Artefakt, Störung und Krise haben wir im Projektkontext zunächst jeweils für alle drei Phänomene eigenständige Raster angelegt. Für Störungen wurde das angelegte Raster nach einiger Zeit noch einmal in «Störungsauftreten» und «Störungsbearbeitung» sowie für Krisen in "Krisenauftreten» und «Krisenbearbeitungsversuche» differenziert. Diese Raster enthalten jeweils eigene Sequenzen, die untereinander sowie mit den anderen Rastern verglichen werden können. Man kann damit anfangen, ein Raster für ein Datenstück anzufertigen und dann später dazu übergehen, mehrere Datenstücke unter einer Fragestellung in einem Raster zusammenzufassen.

Die hier vorgeschlagenen analytischen Dimensionen (Device, Körper, Verbalsprache, externe Komplexität) sind - je nach Forschungsfokus und Fragestellung - austauschbar; sie variieren mit Fragestellung und Gegenstand. So wäre es z. B. vorstellbar - je nach Material -, die Dimension «Verbalsprache» weiter in unterschiedliche sprachliche oder erzählerische Stilmittel und Gattungen zu differenzieren, oder auch die Analysedimension «Körper» beispielsweise nach unterschiedlichen Posituren oder nach dem Aufkommen von «gesture phases» (Kendon 2004) weiter zu differenzieren. Es ist zudem unverzichtbar, parallel zum Anlegen eines Analyserasters extensivere (multimodale) Transkripte oder Partituren einiger schon analysierter Sequenzen anzufertigen. In diesen Transkripten wird dann realisiert, was weiter oben unter 3. als "Auswahl von zu transkribierenden Modalitäten» bezeichnet wurde. Hier werden dann vor allem mittels technischer Brechungen der temporalen Struktur des Datenmaterials - das ist das (methodo)logische Komplementärstück zu dem was hier

16 Der sequenziellen Logik zu folgen heisst dann auch, die prinzipielle Kontingenz von Anschlussmöglichkeiten durchzuspielen, um so zu ermitteln, warum in einer bestimmten Weise, an eine bestimmte Sequenzstelle, an einen vorausgehenden Redezug oder eine Aktivität angeschlossen wird oder auf welchen vorausgegangenen Sachverhalt eine bestimmte Aktivität eine «Antwort» gibt und was ihr folgt. 
«Echtzeitanalyse in Gruppensettings» genannt wurde - feine körper-leibliche Bezugsmomente der jeweiligen Protagonistinnen und Protagonisten sichtbar gemacht, die u. E. insbesondere für die Analyse von dis/ability äusserst instruktiv sind.

\section{Dis/ability}

Heath, Hindmarsh und Luff betonen, "[a]udio-visual recordings are increasingly used to support research that examines the situated activities and interactional organisation through which knowledge, skills and practice are shared and disseminated» $(2010,8)$. Daran anknüpfend gehen wir davon aus, dass sich Analysen von audio-visuellen Daten besonders gut dazu eignen, die zumeist impliziten Mechanismen ableistischer Praktiken aufzudecken. Ableism steht für

«eine analytische Perspektive, die innerhalb des US-amerikanischen Disabled People's Movement sowie der Disability Studies entwickelt wurde, um eine kritische Auseinandersetzung mit fähigkeitsbezogenen Regimen, über die bestimmte Normalitäten, Zwänge und Ausschlüsse hergestellt werden, zu befördern. Dabei wird der Blick für einen besonderen Produktionsmodus sozialer Ungleichheit geschärft, der über die An- und Aberkennung von Fähigkeiten organisiert wird» (Buchner und Lindmeier 2019, 234).

In diesem Zusammenhang legt unser Datenmaterial nahe, dass «abilities» und komplementär dazu «disabilities» in situ dynamisch hervorgebracht werden und eben nicht als invariante und vorsituative Grössen zugrunde gelegt werden können. Demnach verstehen wir unter dis/ability einen Interaktionsprozess in einem sozio-materiellen Netzwerk, in dem abilities und disabilities dynamisch vermittelt (bzw. übersetzt) werden. Man würde demnach erst einmal nicht von fixen Positionen ausgehen, die sich im Zeitverlauf nicht verändern, sondern davon, dass wer gerade (wodurch) abled oder disabled ist im Zeitverlauf variiert. Wer oder was gerade abled oder disabled ist, wird so zu einer empirischen Frage, die am besten anhand 
der Analysen von audio-visuellem Material geklärt werden kann. Zudem ist für uns eine Perspektive anschlussfähig, die Schillmeier als «Rethinking Disability» bezeichnet:

«Rethinking Disability advocates focusing on the heterogeneity of very local - enabling and disabling - practices that are always more complex than any global perspective of them.» (2010, 182).

Hier wird dann eine «prozessuale Dimension des Sozialen» in den Vordergrund gerückt, die erst durch materiale Komplexitäten «ihren ereignishaften Charakter ermöglicht und erhält» (ebd. 2007, 84). Dis/ability verweist dann auf «das komplexe Zusammenspiel von Körpern, Sinnen, Gefühlen, Symbolen, Erfahrungen, Technologien [...]» (ebd., 91) usw. Um diesem Zusammenspiel auf die Spur zu kommen, wurde im Rahmen des Beitrags bereits vorgeschlagen, audio-visuelle Daten zugrunde zu legen und sie zunächst mittels der Trias «Artefakt, Störung, Krise» zu analysieren. Artefakte sind im Rahmen unserer Überlegungen in erster Linie produktive Körper- und Leibesphänomene. Für den Kontext dis/ability treten sie zumeist spontan und punktuell auf und entziehen sich der Steuerbarkeit durch die Beteiligten. Für Störungen gilt, dass sie in der Regel antizipierbar sind. Den Interaktionsteilnehmenden steht dann ein Repertoire an Routinen zur Verfügung, das es ihnen ermöglicht, diese Störungen unmittelbar zu bearbeiten. Für den Kontext dis/ability gilt, dass «Störungen» in alltagsweltlichen Kommunikationssituationen - möglicherweise nicht so routinehaft bearbeitet werden können. Fällt beispielsweise ein Talker - ein (elektronisches) Gerät für die unterstützte Kommunikation - aus, bedarf es Sachkenntnis und Zeit, dieses Gerät wieder zum Laufen zu bringen. Aufgrund dieser komplexen Voraussetzungen kann es somit auch eben potenziell schneller zu temporären oder auch dauerhaften Krisen kommen, da (unmittelbare) Ressourcen zur Störungsbearbeitung und Krisenabwehr nicht vorhanden sind.

Zudem gilt für den Kontext dis/ability, dass Menschen mit Stigmata gekennzeichnet werden. Goffman (1975) geht davon aus, dass ein Stigma in der Divergenz von bestimmten (virtualen) Vorannahmen von einer Person im Hinblick auf eine andere (oder einen Personenkreis) auf der einen und den aktualen situativen und von einer Person «bezeugten» Gegebenheiten 
auf der anderen Seite kondensiert (vgl. ebd. 1975, 10). Ein Stigma wäre demnach zunächst die Zuschreibung eines negativen Attributs zur persönlichen Identität eines Menschen. ${ }^{17}$ Goffman unterscheidet weiter zwischen drei Typen von Stigma: Körperstigmata (1), Charakterstigmata (2) und Phylogenetische Stigmata (vgl. ebd. 12). Erstere betreffen körperliche Merkmale, zweitere psychologische Dispositionen und letztere die Generalisierung und Naturalisierung von Stigmata mit Blick auf (intergenerationale) Gruppen (bspw. Familien). Aus der hier eingenommenen Perspektive sind die Ausführungen Goffmans zwar hilfreich, u. E. reicht es aber nicht aus, im Kontext von dis/ability von vornehmlich individualistisch und asymmetrisch konzeptualisierten «Zuschreibungen» zu sprechen. Wir plädieren hingegen dafür, dem situativen Zusammenspiel von sozio-materialen und sozio-technischen Komplexitäten Rechnung zu tragen.

\section{Resümee}

Wie wir versucht haben zu zeigen, ist Artefakten, Störungen und Krisen gemein, dass sie nicht einfach «nach Plan» auftreten, sondern in komplexen (sozio-materiellen) Situationen emergieren. ${ }^{18}$ Im Rahmen des hier vorgestellten Forschungskontexts wurde danach gefragt, wie Artefakte, Störungen und Krisen konstitutionspraktisch miteinander zusammenhängen. Können Krisen beispielsweise durch «zu viel» oder «zu wenig» Störung oder Artefakte entstehen? Es gilt - jeweils empirisch -, das Verhältnis der drei Phänomene zueinander zu klären. Die Begriffe sind dabei in Auseinandersetzung mit dem Material unter Zuhilfenahme des Analyserasters entstanden und wurden - am Material - weiter analytisch präzisiert. Sie

17 Goffman unterscheidet zwischen ich-Identität, personaler Identität und sozialer Identität. Letztlich würde das Stigma dieser Logik nach als Attribut der personalen Identität konstitutiv und weiter in die «Ich-Identität» einer Person diffundieren und damit auch in der sozialen Identität verankert. Für den Prozess des Stigmatisierens gilt dann letztlich, dass ein virtuales Stigma aktual bestätigt, aber eben auch (aktual) widerlegt werden kann. Aktual ist es wiederum auch möglich, virtuale (Vor-)urteile zu kondensieren und zu generalisieren.

18 In unserem Datenmaterial lässt sich auch eine Art Gegenstück zu einer Krise finden. Dieses Phänomen lässt sich im Kontext von Musizierpraktiken als eine Art Synchronisationsaktivität, als subtile Rhythmusbewegungen, bzw. als (gemeinsamer) Einschwingvorgang identifizieren. 
weisen dabei in unterschiedliche Analyserichtungen. Artefakte sollen in erster Linie die Bezugsgrösse Körper in Rechnung stellen, Störungen (technische) Geräte und Krisen (kommunikative) Macht.

Für die im Rahmen des Forschungsprojekts erzeugten Daten lässt sich sagen, dass Krisen selten, Artefakte und Störungen dagegen häufig auftreten. Artefakte und Störungen sind zudem die Ereignisse, die sich - in unserem Forschungsfeld - am eindeutigsten analytisch identifizieren lassen. Empirisch gesehen treten Artefakte und Störungen mit einer gewissen Regelmässigkeit in allen (natürlichen) Interaktionssituationen auf. Man kann in diesem Zusammenhang die methodologische Prämisse vorbringen, dass sich Interaktionen und ihre Krisen nicht jenseits von körperlich-dinglichen Praktiken beobachten lassen und deshalb eine Methode diesem Sachverhalt Rechnung tragen muss. Einerseits warten Krisen mit dem Potenzial auf, etablierte Machtasymmetrien punktuell ausser Kraft zu setzen, andererseits vermögen sie es, interaktionale Zusammenhänge dauerhaft zu sprengen, was zumeist mit negativen sozialen, körperlichen und psychischen Konsequenzen einhergeht.

\section{Literatur}

Antony, Alexander, Gerd Sebald, und Frank Adloff. 2016. «Handlungs- und Interaktionskrisen: Eine Annährung in systematisierender Absicht». Österreichische Zeitschrift für Soziologie. Sonderheft 1, Handlungs- und Interaktionskrisen, herausgegeben von Frank Adloff, Alexander Antony, und Gerd Sebald, 1-15. Wiesbaden: Springer VS. https://doi.org/10.1007/s11614-016-0209-7.

Apel, Heiner, Andreas Corr, und Anna Valentine Ullrich. 2013. «Produktive Störungen: Pause, Schweigen, Leerstelle». In Das 〈Prinzip Störung〉 in den Geistesund Sozialwissenschaften - Studien und Texte zur Sozialgeschichte der Literatur, Band 133, herausgegeben von Carsten Gansel und Norman Ächtler, 97-112. Berlin: de Gruyter. https://doi.org/10.1515/9783110314076.

Baecker, Dirk. 2011. «Organisation und Störung: Aufsätze». In Wissenschaft 2012, herausgegeben von Dirk Baecker, Berlin: Suhrkamp.

Bateson, Gregory. 1969. «Double bind». In Steps to an ecology of the mind, herausgegeben von Gregory Bateson, 271-278. New York: Ballantine.

Bergmann, Jörg. 2012. Irritationen, Brüche, Katastrophen - Über soziale Praktiken des Umgangs mit «Störungen» in der Interaktion. Bielefeld: Universität Bielefeld. https://www.uni-bielefeld.de/fakultaeten/soziologie/fakultaet/personen/emeriti/bergmann/publikationen/pdf/Bergmann_2012-Abschiedsvorlesung.pdf. 
Breuer, Franz, Barbara Dieris, und Petra Muckel. 2019. Reflexive Grounded Theory: Eine Einführung für die Forschungspraxis. Wiesbaden: Springer VS. https://doi. org/10.1007/978-3-658-22219-2.

Buchner, Tobias, und Christian Lindmeier. 2019. "Zur Einführung: Grundzüge, Rezeptionslinien und Desiderate ableismuskritischer Forschung im deutschsprachigen Raum». Sonderpädagogische Förderung heute 3: 233-239.

Corbin, Juliet M., und Anselm L. Strauss. 2015. Basics of qualitative research: techniques and procedures for developing grounded theory. 4. Aufl. Los Angeles: Sage.

Gansel, Carsten. 2013. «Zu Aspekten einer Bestimmung der Kategorie 〈Störung〉 - Möglichkeiten der Anwendung für Analysen des Handlungs- und Symbolsystems Literatur». In Das 〈Prinzip Störung〉 in den Geistes- und Sozialwissenschaften - Studien und Texte zur Sozialgeschichte der Literatur, Band 133, herausgegeben von Carsten Gansel und Norman Ächtler, 31-56. Berlin: de Gruyter. https://doi.org/10.1515/9783110314076.31.

Gansel, Carsten, und Norman Ächtler. 2013. «Das 〈Prinzip Störung〉 in den Geistes- und Sozialwissenschaften. Einleitung». In Das 〈Prinzip Störung〉 in den Geistes- und Sozialwissenschaften - Studien und Texte zur Sozialgeschichte der Literatur, Band 133, herausgegeben von Carsten Gansel und Norman Ächtler, 7-13. Berlin: de Gruyter. https://doi.org/10.1515/9783110314076.

Geertz, Clifford. 2017. The interpretation of cultures: selected essays. New York: Basic Books.

Glaser, Barney G., Strauss, Anselm L. 2017. The discovery of grounded theory: strategies for qualitative research. London and New York: Routledge, https://doi. org/10.4324/9780203793206.

Goffman, Erving. 1955. «On face-work: an analysis of ritual elements in social interaction». Psychiatry: Journal for the Study of Interpersonal Processes 18: 213231. https://doi.org/10.1080/00332747.1955.11023008.

Goffman, Erving. 1967. Interaction ritual. Essays on face-to-face behavior. New York: Pantheon Books. https://doi.org/10.4324/9780203788387.

Goffman, Erving. 1975. Stigma: über Techniken der Bewältigung beschädigter Identität. Frankfurt am Main: Suhrkamp.

Goodley, Dan. 2017. Disability studies. An interdisciplinary introduction. 2. Aufl. London: SAGE.

Groenemeyer, Axel. 1999. «Soziale Probleme, soziologische Theorie und moderne Gesellschaften». In Handbuch soziale Probleme, herausgegeben von Albrecht Günter und Axel Groenemeyer, 13-72. Wiesbaden: VS Verlag für Sozialwissenschaften. https://doi.org/10.1007/978-3-322-99640-4.

Heath, Christian, Jon Hindmarsh, und Paul Luff. 2011. Video in qualitative research: analysing social interaction in everyday life. Los Angeles: Sage.

Heidegger, Martin. 1953. Sein und Zeit. 7., unveränd. Aufl. Tübingen: Niemeyer. 
Hilgers, Andrea. 1997. Artefakt und empirische Sozialforschung Genese und Analyse der Kritik. Sozialwissenschaftliche Schriften. Berlin: Duncker und Humblot.

Kämpf, Heike. 2018. «Das Subjekt und die Spuren der Macht». In Macht:Denken, herausgegeben von Katrin Felgenhauer, und Falk Bornmüller, 159-174. Bielefeld: transcript. https://doi.org/10.14361/9783839441206.

Kendon, Adam. 2004. Gesture: Visible Action as Utterance. Cambridge University Press.

Knoblauch, Hubert. 2004. «Die Video-Interaktions-Analyse». Sozialer Sinn 5 (1): 123-138. https://nbn-resolving.org/urn:nbn:de:0168-ssoar-7571.

Krämer, Hannes. 2016. «Die Krisen der Ethnomethodologie. Zur Methodologie und Theorie des Disruptiven bei Harold Garfinkel». Österreichische Zeitschrift für Soziologie, Sonderheft 1, Handlungs- und Interaktionskrisen, herausgegeben von Frank Adloff, Alexander Antony, und Gerd Sebald, 35-56. Wiesbaden: Springer VS. https://doi.org/10.1007/s11614-016-0205-y.

Latour, Bruno. 2008. Wir sind nie modern gewesen: Versuch einer symmetrischen Anthropologie. 4. Aufl. Frankfurt am Main: Suhrkamp

Luhmann, Niklas. 1997. «Die Gesellschaft der Gesellschaft». In Die Gesellschaft der Gesellschaft, 1: [Kapitel I - 3], herausgegeben von Niklas Luhmann, Frankfurt am Main: Suhrkamp.

Mannheim, Karl. 1950. Freedom, Power, and Democratic Planning. New York: Oxford University Press. https://doi.org/10.2307/1950904.

Mohn, Bina Elisabeth. 2013. «Differenzen zeigender Ethnographie. Blickschneisen und Schnittstellen der Kamera-Ethnographie». Soziale Welt 64 (1/2): 171189. https://doi.org/10.5771/0038-6073-2013-1-2-171.

Saar, Martin. 2017. «Die Form der Macht». In Vierzig Jahre «Überwachen Und Strafen»: Zur Aktualität der Foucault'schen Machtanalyse, herausgegeben von Roberto Nigro und Marc Rölli, 157-174. Leipzig: transcript. https://doi. org/10.14361/9783839438473.

Schatzki, Theodore. 2016. "Crises and adjustements in ongoing life». Österreichische Zeitschrift für Soziologie. Sonderheft 1, Handlungs- und Interaktionskrisen, herausgegeben von Frank Adloff, Alexander Antony, und Gerd Sebald, 17-33. Wiesbaden: Springer VS. https://doi.org/10.1007/s11614-016-0204-z.

Schillmeier, Michael. 2007. «Zur Politik des Behindert-Werdens. Behinderung als Erfahrung und Ereignis». In Disability studies, Kultursoziologie und Soziologie der Behinderung: Erkundungen in einem neuen Forschungsfeld, herausgegeben von Anne Waldschmidt, Bielefeld: transcript. https://doi. org/10.14361/9783839404867-004.

Schillmeier, Michael. 2010. Rethinking disability. Bodies, Senses, and Things. Routledge studies in science, technology and society 11. New York: Routledge. https://doi.org/10.4324/9780203854846. 
Schmitz, Hermann. 1969. System der Philosophie Band 3, Teil 2: Der Gefühlsraum. Bonn: Bouvier.

Spitta, Julia. 2018. «Der ungebetene Gast. Die Krise des Subjekts zwischen Verdrängung und Wiederkehr». Zeitschrift für Kulturphilosophie 2 (Subjektivierung): 205-432. https://doi.org/10.28937/1000108261.

Strübing, Jörg. 2014. Grounded Theory: Zur sozialtheoretischen und epistemologischen Fundierung eines pragmatistischen Forschungsstils. Wiesbaden: VS Verlag für Sozialwissenschaften. https://doi.org/10.1007/978-3-531-19897-2.

von Foerster, Heinz. 2003. "On Self-Organizing Systems and Their Environments». Understanding Understanding. Essays on Cybernetics and Cognition, New York: Springer. https://doi.org/10.1007/0-387-21722-3_1.

Willke, Helmut. 1994. "Systemtheorie, 2: Interventionstheorie». In Uni-Taschenbücher 1800, Systemtheorie, herausgegeben von Helmut Willke, Stuttgart: G. Fischer. 